\title{
Dynamic Parameters do not Predict Fluid Responsiveness in Ventilated Patients with Severe Sepsis or Septic Shock
}

\author{
Zubair Umer Mohamed ${ }^{1 *}$, Paul Finn'2, Vijay Jagannathan ${ }^{3}$, Sanjiv Sharma ${ }^{4}$ and Jost Mullenheim ${ }^{4}$ \\ ${ }^{1}$ Department of Anaesthesia and Critical Care, Amrita Institute of Medical Sciences and Research Centre, India \\ ${ }^{2}$ Department of ENT, The James Cook University Hospital, United Kingdom \\ ${ }^{3}$ Department of Anaesthesia, University Hospital of North Tees, United Kingdom \\ ${ }^{4}$ Department of Anaesthesia, The James Cook University Hospital, United Kingdom
}

Submission: February 01, 2017; Published:May 30, 2017

*Corresponding author: Zubair Umer Mohamed, Department of Anaesthesia and Critical Care, Amrita Institute of Medical Sciences and Research Centre, India, Tel: 00919496038812; Email: zubairumer@gmail.com

\begin{abstract}
The dynamic parameters, stroke volume variation (SVV) and pulse pressure variation (PPV), are used to guide fluid resuscitation in ventilated patients. We investigated whether SVV, PPV and pleth variability index (PVI), an automatic measurement of the plethysmographic waveform amplitude changes, can be used to predict fluid responsiveness in ventilated patients with severe sepsis or septic shock. We measured cardiac index, (CI, transpulmonary thermodilution PiCCO2) SVV, PPV, global end-diastolic index (GEDI), central venous (CVP), arterial blood pressure and PVI (Masimo Radical 7) before and after infusion of $500 \mathrm{ml}$ Gelofusine ${ }^{\circledR}$ over $30 \mathrm{~min}$ in 31 deeply sedated ventilated patients (tidal volume $8 \mathrm{ml} / \mathrm{kg}$ ) with severe sepsis and septic shock. We obtained one full set of measurements in 30 patients. 10 patients increased CI by at least $15 \%$ ("responders"), 20 patients were "non-responders". Baseline haemodynamic variables were not significantly different between both groups. The area under the receiver operating curves (mean, SE) were 0.68 (0.11) for SVV, $0.66(0.12)$ for PPV, 0.59 (0.12) for PVI, 0.55 (0.12) for GEDI and $0.75(0.09)$ for CVP. We concluded that none of the investigated dynamic parameters could reliably predict fluid responsiveness in ventilated patients with severe sepsis and septic shock in our study.
\end{abstract}

\section{Introduction}

Shock in sepsis results from vasodilatation and a reduction of effective intravascular volume. Its treatment, among others, includes optimal fluid resuscitation. Both over and under resuscitation can worsen outcome in these patients [1]. Routine clinical examination and static indicators of cardiac preload such as central venous pressure (CVP), pulmonary capillary wedge pressure (PCWP), or left ventricular (LV) end diastolic area, are poor predictors of fluid responsiveness [1,2]. Recent studies have shown that respiratory variations in the dynamic indicators of LV stroke volume (SV), namely pulse pressure variation (PPV) and SV variation (SVV) are more reliable predictors of fluid responsiveness in ventilated septic patients [3-5]. Respiratory changes in the amplitude of the plethysmographic pulse wave $(\triangle \mathrm{POP})$ have been shown to be as accurate as PPV in predicting fluid responsiveness in ventilated septic patients [5]. Pleth variability index (PVI), an automatic and continuous monitor of $\triangle \mathrm{POP}$, has been demonstrated to predict fluid responsiveness in ventilated patients undergoing general anaesthesia [6], and in critically ill ventilated patients with circulatory insufficiency [4]. However, it is unclear whether PVI specifically predicts fluid responsiveness in ventilated patients with severe sepsis or septic shock. Therefore, we conducted a prospective, non-randomised, nonblinded observational study to compare the ability of multiple dynamic and static cardiovascular parameters to predict fluid responsiveness in mechanically ventilated patients with severe sepsis or septic shock.

\section{Materials and Methods}

The study protocol for this observational study was approved by both national and local ethics committees and was conducted in accordance with the Declaration of Helsinki of the World Medical Association. A valid informed and written consent was obtained from patients' next of kin, after detailed explanation 
of the protocol, prior to enrolment into the study. Retrospective consent was obtained from all patients who survived to discharge from intensive care and regained mental capacity.

\section{Patients}

Thirty-one adult non-pregnant patients who required sedation and controlled mechanical ventilation for treatment of severe sepsis or septic shock, as defined by the International Sepsis Definitions Conference [7], were enrolled in the study. Patients were subjected to a fluid challenge $(500 \mathrm{ml}$ of Gelofusine $\AA$ administered over $30 \mathrm{~min}$ ) if they showed at least one sign of inadequate tissue perfusion (systolic blood pressure less than $90 \mathrm{mmHg}$, urine output less than $0.5 \mathrm{mlkg}$ 1h-1 for more than 2 hours, tachycardia greater than 100 beats per minute or capillary refill greater than 2 seconds). Patients were sedated with a continuous infusion of Protocol and Alfentanil. Infusions were titrated to achieve a Richmond Agitation Sedation Scale of -3. Patients were ventilated with a pressure controlled mode (BIPAP mode, EVITA 4 XL, Draeger, Germany) with a tidal volume of $8 \mathrm{ml} / \mathrm{kg}$ estimated ideal body weight and a positive end-expiratory pressure of not more than $15 \mathrm{~cm} \mathrm{H20.} \mathrm{Respiratory} \mathrm{rate} \mathrm{was} \mathrm{adjusted} \mathrm{to} \mathrm{achieve} \mathrm{an} \mathrm{arterial}$ partial pressure of $\mathrm{CO} 2$ of $4.8-6 \mathrm{kPa}$. The $\mathrm{FiO} 2$ was titrated to achieve an arterial saturation of $>92 \%$, the ratio of inspiratory versus expiratory time did not exceed 1:1. Exclusion criteria included any spontaneous breathing activity, a known allergy to Gelofusine $\AA$, any cardiac rhythm other than sinus rhythm, contraindications for a fluid challenge $(\mathrm{PaO} 2 / \mathrm{FiO} 2$ less than $13.3 \mathrm{kPa}$, pulmonary oedema on chest X-ray), patients unable to lie supine or peripheral vasoconstriction causing obliteration of the plethysmographic signal.

\section{Haemodynamic monitoring}

Invasive haemodynamic monitoring was performed by using either a $20 \mathrm{~cm} 5$-Fr thermistor-tipped arterial thermodilution catheter (Pulsiocath, Pulsion Medical Systems AG, Germany) inserted into a femoral artery or by using a $22 \mathrm{~cm} 4-\mathrm{Fr}$ thermistor-tipped arterial thermodilution catheter (Pulsiocath, Pulsion Medical Systems AG, Germany) inserted into a brachial artery. The tip of a central venous catheter (Arrow International Inc., Reading, PA, USA) was positioned in the superior cava vein confirmed by X-ray examination. Central venous blood gas samples were taken pre and post fluid challenge (ABL 725, Radiometer, Copenhagen, Denmark). The arterial catheter was connected to an advanced haemodynamic monitor (PiCCO2 $®$, Pulsion Medical Systems AG, Munich, Germany). Thermodilution was performed using at least three cold fluid boluses randomly throughout the respiratory cycle and was repeated within five minutes prior to and five minutes post fluid administration. The patient was positioned supine for all measurements. Electrocardiogram, arterial blood pressure, CVP and arterial oxygen saturation (SaO2) were continuously monitored (Spectrum Monitor, Datascope Corporation, Montvale, NJ, USA) and all recordings were taken at end-expiration. A pulse oximeter probe (LNCS® Adtx, Masimo Corp., USA) was attached to the index finger of the right hand and wrapped to prevent outside light from interfering with the signal. This pulse oximeter probe was connected to the Masimo Radical 7 monitor (Masimo SET, Masimo Corp., Irvine, CA, USA) displaying perfusion index and Pleth Variability Index (PVI).

\section{Conduct of the study}

After ensuring at least a 5-minute period of haemodynamic stability, the first set of measurements was obtained. This was followed by a fluid bolus of $500 \mathrm{ml}$ Gelofusine $\AA$ infused intravenously over $30 \mathrm{~min}$. The second set of measurements was obtained $5 \mathrm{~min}$ after the fluid infusion was completed. Ventilator settings and dosages of inotropic, vasoactive and anaesthetic drugs were held constant throughout the measurements. At each step of the protocol, the following variables were recorded: Heart rate (HR), systolic, diastolic and mean arterial pressure (MAP), CVP, central venous oxygen saturation (Scv02), SV, SV index (SVI), CO, cardiac index (CI), global end-diastolic index (GEDI), Sp02, PPV, SVV and PVI. All patients were kept in a supine position during the entire period of the study. Only one full set of data was obtained and analysed per patient.

\section{Statistics}

In accordance with previous studies [8], we took the criteria of a $15 \%$ increase in CI in response to the fluid challenge to differentiate responders from non-responders to fluid. The normality of distribution of data was tested using the Kolmogorov-Smirnov test. Parametric data are presented as mean with standard deviation or standard error and nonparametric data as median with inter-quartile range (IQR).

We compared non-parametric haemodynamic data before and after volume expansion in responder and non-responder patients using the Mann-Whitney U test. Wilcoxon signed rank tests were used to compare the response to fluid in responders and non-responders, respectively. Receiver operating characteristic (ROC) curves comparing the ability of CVP, SVV, PPV, GEDI and PVI at baseline to discriminate between responders and nonresponders to volume expansion were generated varying the discriminating threshold of each parameter. Using the results from previously published studies [3], we conducted a priori power calculation which showed that 31 patients were necessary to detect differences of 0.1 between areas under the ROC curves with a $5 \%$ two-sided type I error and $80 \%$ power. A p-value less that 0.05 was considered as significant. All statistical analyses were performed using IBM SPSS Statistics for Windows, Version 20.0 .

\section{Results}

Thirty-one patients were recruited. One patient declined to provide consent retrospectively. Complete sets of data were analysed for the remaining 30 patients. Baseline characteristics, as well as respiratory variables and vasopressor/inotropic 
requirements were not statistically different between responders and non-responders (Table 1). Ten patients increased CI by $15 \%$ or more after volume expansion and were classified as responders. 20 patients were classified as nonresponders. There was no statistically significant difference in any haemodynamic variable at baseline between the two groups (Table 2). Both responders and non-responders increased CVP and decreased PPV in response to the fluid challenge (Table 3 \&
4). Only responders showed a statistically significant increase in GEDI (Table 3). Receiver operating characteristic curves (ROC) comparing the ability of CVP, SVV, PPV, PVI and GEDI to predict fluid responsiveness is shown in (Figure 1). The area under the receiver operating curves (mean, SE) were $0.68(0.11)$ for SVV, 0.66 (0.12) for PPV, 0.59 (0.12) for PVI, 0.55 (0.12) for GEDI and $0.75(0.09)$ for CVP (Table 5, Figure 1).

Table 1: Patient characteristics and baseline respiratory variables presented as mean and standard deviation (SD) or number, unless specified.

\begin{tabular}{|c|c|c|c|}
\hline Parameter & Responders (n=10) & Non-Responders $(n=20)$ & $\mathbf{p}$ \\
\hline Age (years) & $59.3(12.8)$ & $56.6(16.5)$ & 0.65 \\
\hline $\operatorname{Sex}(M / F)$ & 3-Jul & 9-Nov & 0.69 \\
\hline Height $(\mathrm{cm})$ & $172.1(10.83)$ & $170.45(8.06)$ & 0.47 \\
\hline Weight (Kg) & $80(6.6)$ & $75.15(12.43)$ & 0.26 \\
\hline BSA (m2) & $2.02(0.1)$ & $1.93(0.2)$ & 0.23 \\
\hline Source of sepsis (Chest/Abdomen/Others) & $6 / 2 / 2002$ & $10 / 8 / 2002$ & 0.57 \\
\hline \multicolumn{4}{|l|}{ Inotropes and vasopressors } \\
\hline Noradrenaline $\left(\mathrm{mcg} \mathrm{kg}^{-1} \mathrm{~min}^{-1}\right)$ & $0.65(0.63)$ & $0.63(0.59)$ & 0.97 \\
\hline Dobutamine $\left(\mathrm{mcg} \mathrm{kg}^{-1} \mathrm{~min}^{-1}\right)$ & $0.84(1.84)$ & $1.25(4.04)$ & 0.77 \\
\hline Vasopressin $\left(\mathrm{iu} \mathrm{hr}{ }^{-1}\right)$ & ---- & 2.4 & ---- \\
\hline Adrenaline $\left(\mathrm{mcg} \mathrm{kg}^{-1} \mathrm{~min}^{-1}\right)$ & --- & 0.88 & --- \\
\hline $\mathrm{FiO}_{2}$ & $0.54(0.14)$ & $0.57(0.22)$ & 0.66 \\
\hline Peak Inspiratory Pressure $\left(\mathrm{cm} \mathrm{H}_{2} \mathrm{O}\right)$ & $24.4(7.2)$ & $22.65(5.3)$ & 0.46 \\
\hline PEEP $\left(\mathrm{cm} \mathrm{H}_{2} \mathrm{O}\right)$ & $9.5(3.27)$ & $9.35(3.1)$ & 0.9 \\
\hline Mean Airway Pressure $\left(\mathrm{cm} \mathrm{H}_{2} \mathrm{O}\right)$ & $16(4.52)$ & $15.05(4.3)$ & 0.59 \\
\hline Tidal Volume (ml) & $546.1(97.78)$ & $580.75(120.66)$ & 0.44 \\
\hline $\mathrm{PaO}_{2}(\mathrm{KPa})$ & $13.47(4.84)$ & $15.5(4.91)$ & 0.29 \\
\hline $\mathrm{PaO}_{2} / \mathrm{FiO}_{2}$ & $27.02(12.17)$ & $30.44(12.66)$ & 0.49 \\
\hline Dynamic compliance $\left(\mathrm{ml} \mathrm{cm} \mathrm{H} \mathrm{H}_{2} \mathrm{O}^{-1}\right)$ & $50.56(52.65)$ & $47.22(16.65)$ & 0.8 \\
\hline
\end{tabular}

BSA: Body Surface Area; FiO2 7 Fraction of Inspired Oxygen; PEEP Peak End Expiratory Pressure; PaO2 Partial Pressure of Arterial Oxygen; $\mathrm{PaO} \neg 2 / \mathrm{FiO} 2$ Ratio of Partial Pressure of Arterial Oxygen with Fraction of Inspired Oxygen. Vasopressin and Adrenaline was used only in one patient each.

Table 2: Baseline haemodynamic variables of the responders and non-responders presented as median and inter-quartile range (IQR).

\begin{tabular}{|c|c|c|c|}
\hline Variable & $\begin{array}{c}\text { Responders } \\
(\mathbf{n = 1 0})\end{array}$ & $\begin{array}{c}\text { Non-responders } \\
(\mathbf{n = 2 0})\end{array}$ & $\mathbf{p}$ \\
\hline HR (beats min $\left.{ }^{-1}\right)$ & $91(85-106)$ & $91(72-99)$ & 0.21 \\
\hline MAP (mmHg) & $83(69-89)$ & $78(69-89)$ & 0.68 \\
\hline CVP (mmHg) & $11(9-12)$ & $14(10-17)$ & 0.15 \\
\hline $\begin{array}{c}\text { SVRI (dynes sec } \\
\left.\text { cm }^{-5} \mathrm{~m}^{-2}\right)\end{array}$ & $1443(1264-1876)$ & $1669(1174-1798)$ & 0.97 \\
\hline GEDI (ml m-2) & $708(621-882)$ & $714(675-834)$ & 0.95 \\
\hline CI (l min $\left.{ }^{-1} \mathrm{~m}^{-2}\right)$ & $3.7(2.6-4.3)$ & $3.5(3.0-4.3)$ & 0.78 \\
\hline PPV (\%) & $11.5(8.3-15.8)$ & $8.5(5-10.8)$ & 0.07 \\
\hline SVV (\%) & $14(10.5-19)$ & $10(5-14)$ & 0.13 \\
\hline PVI & $13(10.5-19)$ & $11.5(6.5-16.3)$ & 0.35 \\
\hline ScV02 (\%) & $78.0(71.3-81.0)$ & $79.7(74.1-84.3)$ & 0.38 \\
\hline
\end{tabular}

HR: Heart Rate; MAP: Mean Arterial Pressure; CVP: Central Venous Pressure; SVRI: Systemic Vascular Resistance Index; GEDI: Global End Diastolic Index; CI: Cardiac Index; PPV: Pulse Pressure Variation;
SVV: Stroke Volume Variation; PVI: Pleth Variability Index; ScvO2, central venous oxygen saturation

Table 3: Haemodynamic variables of responders before and after fluid challenge, presented as median and inter-quartile range (IQR).

\begin{tabular}{|c|c|c|c|}
\hline Variable & Before & After & $\mathbf{p}$ \\
\hline HR (beats min $\left.^{-1}\right)$ & $91(72-99)$ & $85(71-95)$ & 0.42 \\
\hline MAP (mmHg) & $78(69-89)$ & $81(74-95)$ & 0.11 \\
\hline CVP (mmHg) & $14(10-17)$ & $16(13-22)$ & $<0.01$ \\
\hline $\begin{array}{c}\text { SVRI (dynes sec } \\
\left.\text { cm }^{-5} \mathrm{~m}^{-2}\right)\end{array}$ & $\begin{array}{c}1669(1174- \\
1798)\end{array}$ & $1410(1038-1823)$ & 0.85 \\
\hline GEDI (ml m$\left.)^{-2}\right)$ & $714(675-834)$ & $758(687-873)$ & 0.19 \\
\hline CI (l min $\left.{ }^{-1} \mathrm{~m}-2\right)$ & $3.5(3.0-4.3)$ & $3.6(3.1-4.6)$ & 0.02 \\
\hline PPV (\%) & $8.5(5-10.8)$ & $6.5(4-9.3)$ & 0.01 \\
\hline SVV (\%) & $10(5-14)$ & $8(6-11.8)$ & 0.02 \\
\hline PVI & $11.5(6.5-16.3)$ & $9.5(6-12.8)$ & 0.16 \\
\hline ScV02 (\%) & $79.7(74.1-84.3)$ & $78.8(70.7-83.9)$ & 0.12 \\
\hline
\end{tabular}


HR: Heart Rate; MAP: Mean Arterial Pressure; CVP: Central Venous Pressure; SVRI: Systemic Vascular Resistance Index; GEDI: Global End Diastolic Index; CI: Cardiac Index; PPV: Pulse Pressure Variation; SVV: Stroke Volume Variation; PVI: Pleth Variability Index; ScvO2, central venous oxygen saturation

Table 4: Haemodynamic variables of non-responders before and after fluid challenge, presented as median and inter-quartile range (IQR).

\begin{tabular}{|c|c|c|c|}
\hline Variable & Before & After & $\mathrm{p}$ \\
\hline HR (beats min $\left.{ }^{-1}\right)$ & $91(72-99)$ & $85(71-95)$ & 0.42 \\
\hline MAP (mmHg) & $78(69-89)$ & $81(74-95)$ & 0.11 \\
\hline CVP (mmHg) & $14(10-17)$ & $16(13-22)$ & $<0.01$ \\
\hline $\begin{array}{c}\text { SVRI (dynes sec } \\
\left.\mathrm{cm}^{-5} \mathrm{~m}^{-2}\right)\end{array}$ & $1669(1174-1798)$ & $1410(1038-1823)$ & 0.85 \\
\hline GEDI (ml m$\left.{ }^{-2}\right)$ & $714(675-834)$ & $758(687-873)$ & 0.19 \\
\hline CI $\left(1 \mathrm{~min}^{-1} \mathrm{~m}^{-2}\right)$ & $3.5(3.0-4.3)$ & $3.6(3.1-4.6)$ & 0.02 \\
\hline PPV (\%) & $8.5(5-10.8)$ & $6.5(4-9.3)$ & 0.01 \\
\hline SVV (\%) & $10(5-14)$ & $8(6-11.8)$ & 0.02 \\
\hline PVI & $11.5(6.5-16.3)$ & $9.5(6-12.8)$ & 0.16 \\
\hline ScvO2 (\%) & $79.7(74.1-84.3)$ & $78.8(70.7-83.9)$ & 0.12 \\
\hline
\end{tabular}

HR: Heart Rate; MAP: Mean Arterial Pressure; CVP: Central Venous Pressure; SVRI: Systemic Vascular Resistance Index; GEDI: Global End Diastolic Index; CI: Cardiac Index; PPV: Pulse Pressure Variation; SVV: Stroke Volume Variation; PVI: Pleth Variability Index; ScvO2, central venous oxygen saturation.

Table 5: Area under the receiver operating curves (ROC) curves, standard error and $95 \%$ confidence intervals of different haemodynamic variables to predict fluid responsiveness.

\begin{tabular}{|c|c|c|c|}
\hline Parameter & AUC & SE & 95\% CI \\
\hline CVP & 0.754 & 0.093 & 0.556 to 0.896 \\
\hline SVV & 0.681 & 0.114 & 0.479 to 0.843 \\
\hline PPV & 0.664 & 0.115 & 0.461 to 0.830 \\
\hline PVI & 0.594 & 0.119 & 0.393 to 0.774 \\
\hline GEDI & 0.547 & 0.117 & 0.349 to 0.734 \\
\hline
\end{tabular}

AUC: Area Under the Curve; SE: Standard Error; Cl: Confidence Interval; CVP: Central Venous Pressure; SVV: Stroke Volume Variation; PPV: Pulse Pressure Variation; PVI: Pleth Variability Index; GEDI: Global End Diastolic Index.

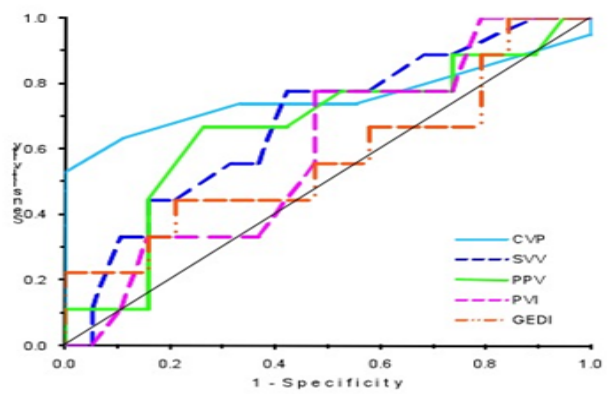

Figure 1: Receiver operating curves for CVP, central venous pressure; SVV, stroke volume variation; PPV, pulse pressure variation; PVI, pleth variability index; GEDI, global end diastolic index.

\section{Discussion}

This study aimed to compare the ability of PVI with the more established parameters PPV, SVV, and GEDI to predict fluid responsiveness in mechanically ventilated patients with severe sepsis or septic shock. The main finding is that none of the above haemodynamic parameters were able to reliably predict fluid responsiveness despite exclusion of common known confounding factors. We observed a significant number of false positive and false negative results considering previously cited cut-off values for dynamic parameters in general ICU and more specifically in ventilated septic patients [4,5,8-10]. Our study population consisted of ventilated patients with severe sepsis and septic shock. All but three patients were receiving vasopressor support. Known confounding variables affecting the ability of dynamic parameters to predict fluid responsiveness were excluded: all patients were in sinus rhythm during the study period and did not have any arrhythmia; all were deeply sedated without any spontaneous breathing activity and received a tidal volume of $8 \mathrm{ml} / \mathrm{kg}$ estimated lean body weight. Haemodynamic measurements were performed using the PiCCO 2 monitor which is a well validated accurate monitor measuring SV even in rapidly changing circulatory conditions [11] and in patients with reduced cardiac function [9]. At least three cold boluses were given randomly throughout the respiratory cycle using the same sampling period (30 seconds) to obtain relevant haemodynamic data using transpulmonary thermodilution [12]. In line with other studies, we used a fluid bolus of $500 \mathrm{ml}$ Gelofusine $\AA$ administered over 30min [5]. The mean CVP increased after volume expansion in both responders and non-responders by at least $2 \mathrm{mmHg}$ (Table $3 \& 4$ ), which has been defined previously as a proof for an adequate fluid challenge [13]. We explored the possible reasons for the unexpected finding that none of the dynamic parameters reliably predicted fluid responsiveness in our study. Less than $50 \%$ of our patients were responders. This is not uncommon in critically ill patients with severe sepsis/septic shock or after cardiac surgery $[10,14,15]$. It is known that septic shock is frequently associated with biventricular dysfunction and increased pulmonary artery pressure [16]. Both RV and LV failure are well known confounders altering the magnitude and ability of PPV and SVV to predict fluid responsiveness [17]. Impaired RV function is also a frequent problem in ARDS, a condition commonly associated with septic shock [18]. In case of RV dysfunction/failure, one might observe "false" high PPV and SVV in non-responders as the RV after load, in contrast to preload change, is the major determinant for high PPV and SVV $[14,19]$. This could be further exacerbated by increased pulmonary artery pressure, large tidal volumes and high PEEP $[18,20]$, the latter two of which were present in our study (Table 1). Previous studies on the ability of dynamic parameters to predict fluid responsiveness in septic patients either did not measure pulmonary artery pressure [5], pulmonary artery pressure was not significantly raised [3] or PEEP values were low [10]. In our study, all but three patients received vasopressors, which 
can independently increase pulmonary artery pressure. Daudel and colleagues demonstrated that, in contrast to haemorrhagic shock, in endotoxemic shock with raised pulmonary artery pressure, PPV did not predict fluid responsiveness [19]. A similar conclusion was reached by VanBallmoos who reported a reduced RV ejection fraction in almost half the non-responders and in none of the responders in patients with septic shock or post cardiac surgery [14].

In case of LV dysfunction/failure both PPV and SVV are generally decreased $[3,17]$. However, Mesquida et al have shown that if PPV and SVV are being used for fluid resuscitation in heart failure conditions, the phase relation between airway pressure and the maximal SV and hence PP needs to be determined [17]. If the LV is afterload dependent, one could observe a simultaneous increase in SV and hence PP when intrathoracic pressure increases and thus PPV and SVV might be high without reflecting fluid responsiveness particularly if the tidal volume is high and/or the chest wall is stiff e.g. due to sepsis induced oedema. For the haemodynamic measurements taken by the PiCCO system the phase relation between the change in airway pressure and maximal PP and SV is unknown. PPV and SVV are calculated over a $30 \mathrm{sec}$ rolling period. Reuter et al reported that SVV measured by the PiCCO system is still a reliable marker of fluid responsiveness in LVF with $\mathrm{EF}<35 \%$ [9]. However, in this study the AUC for SVV to predict fluid responsiveness in patients with impaired LV function was 0.76 which was lower than the AUC for SVV to predict fluid responsiveness in a second group of patients with normal LV function (0.88).

Gruenewald et al reported that in animals suffering from stunned myocardium shortly after cardiac arrest all dynamic parameters are unreliable in predicting fluid responsiveness [21]. Wiesenack and colleagues, found no correlation between SVV measured by the PiCCO system and prediction of fluid responsiveness in patients undergoing elective coronary artery bypass surgery, with an ejection fraction $>50 \%$ [22]. In this study the authors speculated that arterial pulse contourderived estimates of SVV are potentially unreliable under positive pressure ventilation. PPV is considered the more sensitive and specific parameter compared to SVV in predicting fluid responsiveness as pressure measurements are usually more accurate than SV measurements. However, in our study neither baseline SVV nor PPV could reliable predict fluid responsiveness. SV and PP are tightly correlated during positive pressure ventilation [17]. The magnitude of PP for any given SV depends on central arterial compliance. Thus, a vasopressor induced reduction in central arterial compliance could lead to large changes in PP and hence PPV even for small changes in SV. The majority of the patients in our study were treated with vasopressors and it is tempting to speculate that this might be a further explanation why some patients were unresponsive to fluids despite high baseline PPV. Furthermore, it is conceivable that a more pronounced inspiratory increase in PP is due to an exaggerated dUp phenomenon in the presence of reduced LV function [8]. which might have contributed to an increase in PPV in non-responders.

As the cyclic changes in RV and LV pre- and after load are dependent on cyclic changes in intraalveolar, intrapleural and hence transpulmonary pressure any factor affecting one or a combination of these would have an impact on all dynamic parameters. Increasing tidal volume directly increases the magnitude for PPV and SVV for any given chest and lung compliance [17]. Intraabdominal pressure affects chest wall compliance and hence intrapleural pressure. In fact, Jacques et al showed that the cut-off values for all dynamic parameters increase significantly if intraabdominal pressure is increased [23]. We did not measure intra abdominal or intrapleural pressure in our study. Respiratory system compliance was not significantly different in both groups. However, we cannot exclude the possibility that differences in transpulmonary pressures induced by the same tidal volumes might have contributed to our findings. Loupec et al showed that PVI reliably predicts fluid responsiveness in critically ill ventilated patients [4]. However, this result has not always been replicated in septic patients treated with vasopressors $[10,15,24]$. One possible explanation for this finding could be that the proportion of septic shock patients was lower in Loupec's study (55\%) than in the other studies $(85 \%, 86 \%)[4,10,15]$.

\section{Conclusion}

We conclude that the dynamic parameters PPV, SVV and PVI may not be able to predict fluid responsiveness in all ventilated patients with severe sepsis or septic shock even after exclusion of already commonly known confounding factors. An assessment of RV and LV function and measurement of intraabdominal or even transpulmonary pressure should be taken into account before interpreting and acting on the values measured. Passive leg raising, as a "reversible" fluid challenge might help to prevent unnecessary and potential harmful fluid loading provided intraabdominal pressure is not increased [25].

\section{Acknowledgement}

Hardware and software for the conduct of the study were supplied by Masimo Corp., Irvine, CA, USA.

The study was supported by a grant from the Research Development Department, The James Cook University Hospital, Middlesbrough, United Kingdom.

\section{References}

1. Marik PE, Cavallazzi R, Vasu T, Hirani A (2009) Dynamic changes in arterial waveform derived variables and fluid responsiveness in mechanically ventilated patients: A systematic review of the literature. Crit Care Med 37(1): 2642-2647.

2. Marik PE, Baram M, Vahid B (2008) Does central venous pressure predict fluid responsiveness? A systematic review of the literature and the tale of seven mares. Chest 134(1): 172-178.

3. Michard F, Boussat S, Chelma D, Anguel N, Mercat A, et al. (2000) Relation between respiratory changes in arterial pulse pressure and 
fluid responsiveness in septic patients with acute circulatory failure. Am J Respir Crit Care Med 162(1): 134-138.

4. Loupec T, Nanadoumgar H, Frasca D, Petitpas F, Laksiri L, et al. (2011) Pleth variability index predicts fluid responsiveness in critically ill patients. Crit Care Med 39(2): 294-299.

5. Feissel M, Teboul JL, Merlani P, Badie J, Faller JP, et al. (2007) Plethysmographic dynamic indices predict fluid responsiveness in septic ventilated patients Intensive Car Med 33(6): 993-999.

6. Cannesson M, Delannoy B, Morand A, Rosamel P, Attof Y, et al. (2008) Does the pleth variability index indicate the respiratory-induced variation in the plethysmogram and arterial pressure waveforms? Anesth Analg 106(4): 1189-1194.

7. Levy MM, Fink MP, Marshall JC, , Abraham E, Angus D, et al. (2003) 2001 SCCM/ESICM/ACCP/ATS/SIS International Sepsis Definitions Conference. Intensive Car Med 29(4): 530-538.

8. Michard F, Teboul JL (2002) Predicting Fluid Responsiveness in ICU Patients: A Critical Analysis of the Evidence. Chest 121(6): 2000-2008.

9. Reuter DA, Kirchner A, Felbinger TW, Weis FC, Kilger E, et al. (2003) Usefulness of left ventricular stroke volume variation to assess fluid responsiveness in patients with reduced cardiac function. Crit Care Med 31(5): 1399-1404.

10. Monnet X, Guerin L, Jozwiak M, Bataille A, Julien F, et al. (2013) Pleth variability index is a weak predictor of fluid responsiveness in patients receiving norepinephrine. Br J Anaesth 110(2): 207-213.

11. Gödje 0, Höke K, Goetz AE, Felbinger TW, Reuter DA, et al. (2002) Reliability of a new algorithm for continuous cardiac output determination by pulse-contour analysis during hemodynamic instability. Crit Care Med 30(1): 52-58.

12. Monnet X, Persichini R, Ktari M, Jozwiak M, Richard C, et al. (2011) Precision of the transpulmonary thermodilution measurements. Critical Care 15(4): R204

13. Madger S (2006) Central venous pressure: A useful but not so simple measurement. Crit Care Med 34(8): 2224-2227.

14. Von Ballmoos MW, Takala J, Roeck M, Porta F, Tueller D, et al. (2010) Pulse-pressure variation and hemodynamic response in patients with elevated pulmonary artery pressure: a clinical study. Critical Care 14(3): R111.

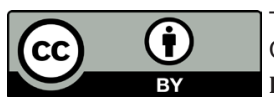

This work is licensed under Creative Commons Attribution 4.0 License DOI:10.19080/JAICM.2017.02.555596
15. Monnet X, Dres M, Ferre A, Le Teuff G, Jozwiak M, et al. (2012) Prediction of fluid responsiveness by a continuous non-invasive assessment of arterial pressure in critically ill patients: comparison with four other dynamic indices. Br J Anaesth 109(3): 330-338.

16. Parker MM, Mc Carthy KE, Ognibene FP, Parrillo JE (1990) Right ventricular dysfunction and dilatation, similar to left ventricular changes, characterize the cardiac depression of septic shock in humans. Chest 97(1): 126-131.

17. Mesquida J, Kim HK, Pinsky MR (2011) Effect of tidal volume, intrathoracic pressure, and cardiac contractility on variations in pulse pressure, stroke volume, and intrathoracic blood volume. Intensive Care Med 37(10): 1672-1679.

18. Jardin F, Delorme D, Hardy A, Auvert B, Beauchet A, et al. (1990) Reevaluation of hemodynamic consequences of positive pressure ventilation: Emphasis on cyclic right ventricular after loading by mechanical lung inflation. Anesthesiology 72: 966 -970.

19. Daudel F, Tüller D, Krähenbühl S, Jakob SM, Takala J (2010) Pulse pressure variation and volume responsiveness during acutely increased pulmonary artery pressure: an experimental study. Critical Care 14(3): R122.

20. Jardin F (2004) Cyclic changes in arterial pressure during mechanical ventilation. Intensive Care Med 30: 1047-1050.

21. Gruenewald M, Meybohm P, Koerner S, Renner J, Maracke M, et al. (2011) Dynamic and volumetric variables of fluid responsiveness fail during immediate postresuscitation period. Crit Care Med 39(8): 1953-1959.

22. Wiesenack C, Prasser C, Rodig G, Keyl C (2003) Stroke volume variation as an indicator of fluid responsiveness using pulse contour analysis in mechanically ventilated patients. Anesth Analg 96(5): 1254 -1257.

23. Jacques D, Bendjelid K, Duperret S, Colling J, Piriou V, et al. (2011) Pulse pressure variation and stroke volume variation during increased intraabdominal pressure: an experimental study. Critical Care 15(1): R33.

24. Landsverk SA, Hoiseth LO, Kvandal P, Hisdal J, Skare O, et al. (2008) Poor agreement between respiratory variations in pulse oximetry photoplethysmographic waveform amplitude and pulse pressure in intensive care unit patients. Anesthesiology 109(5): 849-855.

25. Monnet X, Teboul JL (2008) Passive leg raising. Intensive Care Med 34: 659-663.

\section{Your next submission with Juniper Publishers will reach you the below assets}

- Quality Editorial service

- Swift Peer Review

- Reprints availability

- E-prints Service

- Manuscript Podcast for convenient understanding

- Global attainment for your research

- Manuscript accessibility in different formats

( Pdf, E-pub, Full Text, Audio)

- Unceasing customer service

Track the below URL for one-step submission

https://juniperpublishers.com/online-submission.php 